\title{
An Anti-Neuroinflammatory That Targets Dysregulated Glia Enhances the Efficacy of CNS-Directed Gene Therapy in Murine Infantile Neuronal Ceroid Lipofuscinosis
}

\author{
(1) Shannon L. Macauley, ${ }^{1,2}$ Andrew M.S. Wong, ${ }^{5}$ Charles Shyng, ${ }^{2}$ David P. Augner, ${ }^{5}$ Joshua T. Dearborn, ${ }^{2}$ \\ Yewande Pearse, ${ }^{5}$ Marie S. Roberts, ${ }^{2}$ Stephen C. Fowler, ${ }^{4}$ Jonathan D. Cooper, ${ }^{5}$ D. Martin Watterson, ${ }^{6}$ \\ and Mark S. Sands ${ }^{2,3}$ \\ ${ }^{1}$ Department of Neurology, ${ }^{2}$ Department of Internal Medicine, and ${ }^{3}$ Department of Genetics, Washington University School of Medicine, St. Louis, Missouri \\ 63110, ${ }^{4}$ Departments of Pharmacology and Toxicology, University of Kansas, Lawrence, Kansas 66045, ${ }^{5}$ Department of Neuroscience, Centre for the \\ Cellular Basis of Behaviour, MRC Centre for Neurodegeneration Research, Institute of Psychiatry, King's College London, London SE5 9NU, United \\ Kingdom, and ${ }^{6}$ Department of Pharmacology, Feinberg School of Medicine, Northwestern University, Chicago, Illinois 60611
}

Infantile neuronal ceroid lipofuscinosis (INCL) is an inherited neurodegenerative lysosomal storage disease (LSD) caused by a deficiency in palmitoyl protein thioesterase-1 (PPT1). Studies in $\mathrm{Ppt1}^{-1-}$ mice demonstrate that glial activation is central to the pathogenesis of INCL. Astrocyte activation precedes neuronal loss, while cytokine upregulation associated with microglial reactivity occurs before and concurrent with neurodegeneration. Therefore, we hypothesized that cytokine cascades associated with neuroinflammation are important therapeutic targets for the treatment of INCL. MW01-2-151SRM (MW151) is a blood-brain barrier penetrant, small-molecule anti-neuroinflammatory that attenuates glial cytokine upregulation in models of neuroinflammation such as traumatic brain injury, Alzheimer's disease, and kainic acid toxicity. Thus, we used MW151, alone and in combination with CNS-directed, AAV-mediated gene therapy, as a possible treatment for INCL. MW151 alone decreased seizure susceptibility. When combined with AAV-mediated gene therapy, treated INCL mice had increased life spans, improved motor performance, and eradication of seizures. Combination-treated INCL mice also had decreased brain atrophy, astrocytosis, and microglial activation, as well as intermediary effects on cytokine upregulation. These data suggest that MW151 can attenuate seizure susceptibility but is most effective when used in conjunction with a therapy that targets the primary genetic defect.

Key words: Batten disease; lysosomal storage disease; neurodegeneration; neuroinflammation; neuronal ceroid lipofuscinosis

\section{Introduction}

Infantile neuronal ceroid lipofuscinosis (INCL, or CLN1 disease) is a rare, autosomal recessive lysosomal storage disease that largely affects the CNS beginning in infancy or early childhood (Vesa et al., 1995; Hofmann et al., 1999). INCL is caused by mutations in the CLN1 gene leading to a deficiency in palmitoyl protein thioesterase-1 (PPT1). Typically, patients are asymptomatic at birth and development proceeds normally. However, by 2 years of age, INCL patients suffer from blindness, seizures, cognitive impairment, and motor dysfunction, ultimately leading to death by $4-6$ years of age.

\footnotetext{
Received June 19, 2014; revised Aug. 13, 2014; accepted Aug. 19, 2014
}

Author contributions: S.L.M., J.D.C., and M.S.S. designed research;S.L.M., A.M.S.W., C.S., D.P.A., J.T.D., Y.P., and M.S.R. performed research; S.C.F. and D.M.W. contributed unpublished reagents/analytic tools; S.L.M., A.M.S.W., C.S., D.P.A., J.T.D., Y.P., M.S.R., S.C.F., J.D.C., and M.S.S. analyzed data; S.L.M. and M.S.S. wrote the paper.

This work was supported by the following grants: NS043205 (M.S.S.), NS056728 (S.L.M.), R01NS056051 (D.M.W.), HD002528 (S.C.F.), and a BDSRA postdoctoral research fellowship (S.L.M.).

The authors declare no competing financial interests.

Correspondence should be addressed to Mark S. Sands, Washington University School of Medicine, Department of Internal Medicine, Campus Box 8007, 660 South Euclid Avenue, St. Louis, M0 63110. E-mail: msands@dom.wustl.edu.

DOI:10.1523/JNEUROSCI.2518-14.2014

Copyright $\odot 2014$ the authors $\quad 0270-6474 / 14 / 3413077-06 \$ 15.00 / 0$
Previous studies demonstrate that PPT1-deficient $\left(P p t 1^{-/-}\right)$ mice mimic the clinical features of INCL, such as cognitive, motor, and visual deficits, as well as spontaneous seizures. Interestingly, focal areas of astrocyte activation were the first pathological feature observed in the $\mathrm{Ppt1}^{-1-}$ brain (Kielar et al., 2007; Macauley et al., 2009, 2011; Groh et al., 2013). Coincident with the onset of astrocyte activation was a significant increase in proinflammatory cytokines, microglial activation, and leukocyte recruitment into the brain. Specific brain regions affected by early astrocyte activation were the same regions prone to neurodegeneration and brain atrophy.

Preclinical intervention studies for INCL reveal the promise for enzyme replacement therapy (ERT) via CNS-directed, AAVmediated gene therapy (Griffey et al., 2004, 2006; Macauley et al., 2012; Roberts et al., 2012). However, this approach has proven to be only partially efficacious given the complexity of clinical features associated with INCL. Therefore, isolating secondary disease mechanisms that are responsive to therapeutic intervention is necessary for improved dosing and efficacy. Dosing includes a therapeutic time window, based on pathophysiology progression; frequency of administration, based on how the body reacts to the treatment; as well as the amount of drug administered, 
based on body weight or volume. Therefore, dosing of AAVmediated enzyme replacement to the CNS is a challenge and not readily amenable to the optimization routinely accomplished with small-molecule therapeutics. However, promising replacement therapies can be combined with various complementary treatments based on dosing considerations. Because the enzyme deficiency in the $P p t 1^{-/-}$mice is accompanied by neuroinflammation and increased cytokine production, a rational approach would be to test the potential of an experimental therapeutic such as MW151 as an adjunct therapy. MW151 is a glial-specific, small molecule whose pharmacological mechanism of action is attenuation of injurious upregulation of pro-inflammatory cytokines, which are associated with brain injury, progressive neurodegeneration, or increased pathology susceptibility due to CNS innate immunity priming (Hu et al., 2007; Somera-Molina et al., 2007; Karpus et al., 2008; Chrzaszcz et al., 2010; Bachstetter et al., 2012).

We hypothesized that simultaneous treatment with the small-molecule drug MW151 would improve the outcome of CNS-directed, AAV2/5-mediated gene therapy. In fact, this cotreatment resulted in increased life span, improved motor function, elimination of seizures, and decreased glial activation and cytokine production. The outcomes demonstrate the potential utility of using drugs that attenuate pro-inflammatory cytokine production as a cotherapy for gene-mediated replacement therapy for INCL.

\section{Materials and Methods}

$P p t 1^{-1-}$ and wild-type mice. Congenic wild-type $(+/+)$ or $P p t 1^{-1-}$ $(-/-)$ mice on the C57BL/6 background were generated at Washington University School of Medicine. Genotype was determined by PCR-based assay (Gupta et al., 2001). Male and female $P p t 1^{-1-}$ mice and normal littermates were used. All procedures were performed in accordance with an approved IACUC protocol from Washington University School of Medicine.

Life span. Longevity was assessed in treated $P p t 1^{-1-}$ mice and shamtreated controls ( $n=10-15$ mice/group). End of life was designated by death or a predetermined moribund condition. Kaplan-Meier analysis was used to measure cumulative survival and establish significant differences in life span $(p<0.05)$.

Recombinant $A A V$ production. The rAAV2/5-PPT1 vector used in these studies was generated at the University of Florida Vector Core Laboratory as previously described (Griffey et al., 2004). Briefly, the vector contained the cytomegalovirus enhancer, chicken $\beta$-actin promoter; first intron from the chicken $\beta$-actin gene; human PPT1 cDNA; rabbit $\beta$-globin polyadenylation signal; and flanking inverted terminal repeats from AAV2.

Intracranial injections. On P1, the viral vector was injected intracranially using a Hamilton syringe and 30 gauge needle. Two microliters of virus $\left(1 \times 10^{12} \mathrm{vg} / \mathrm{ml}\right)$ was bilaterally injected into the anterior cortex $(1$ $\mathrm{mm}$ rostral to bregma, $2 \mathrm{~mm}$ mediolateral of midline, and $2 \mathrm{~mm}$ ventral to the surface of the skull), hippocampus/thalamus $(3.5 \mathrm{~mm}$ rostral to bregma, $2 \mathrm{~mm}$ mediolateral of midline, and $2 \mathrm{~mm}$ ventral to the surface of the skull), and cerebellum ( $1 \mathrm{~mm}$ rostral to lambda, $1 \mathrm{~mm}$ mediolateral of midline, and $2 \mathrm{~mm}$ ventral to the surface of the skull).

MW151 injections. A $1 \times$ MW151 solution was made daily in sterile $0.9 \%$ saline, filter sterilized, and immediately injected. Ppt1-deficient mice received daily intraperitoneal injections of MW151 at a dose of 2.5 $\mathrm{mg} / \mathrm{kg}$. Dosing began at $28 \mathrm{~d}$ of age and continued for the remainder of their life. Additionally, separate cohorts of $P p t 1^{-/-}$and WT mice received daily injections of saline and served as controls.

Treatment groups. Five treatment groups were used in this study: (1) WT: WT mice receiving daily saline injections, (2) $P p t 1^{-/-}: P p t 1^{-/-}$mice receiving daily saline injections, (3) MW151: $P p t 1^{-1-}$ mice receiving daily injections of MW151, (4) AAV: Ppt1 ${ }^{-/-}$mice receiving intracranial injections of AAV2/5-PPT1, and (5) AAV + MW151: Ppt1 ${ }^{-/-}$mice receiving both intracranial injections of AAV2/5-PPT1 and daily MW151 injections. Each group contained a total of 20 randomly assigned, mixed-sex mice.

Rotarod testing. Treated and sham-treated mice $(n=10-13$ mice/ group) were tested on the constant-speed rotarod beginning at 4 months of age, each month thereafter as previously described (Macauley et al., 2012). Statistical significance was determined using one-way ANOVAs at each time point followed by post hoc tests.

Seizure monitoring via force plate actometer. A force plate actometer was teamed with simultaneous video monitoring to assess seizure activity within treated and untreated mice at 7.5 months of age. Briefly, a custommade actometer was generated as previously described (Reddy et al., 2011). Simultaneous video monitoring and force plate actometry were gathered for 8 consecutive hours. Detection of a seizure with the actometer was confirmed by visually observing a seizure ("popcorn" seizure followed by freezing) on the video recording during the same time frame. Significant differences in seizure activity between untreated PPT1 ${ }^{-1-}$ mice and treated animals were determined using a $\chi^{2}$ test.

PPT1 activity. At 7.5 months of age, treated and sham-treated mice ( $n=3-6$ ) were killed via lethal injection, the brains bisected sagittally, and the left hemisphere flash frozen in liquid nitrogen. PPT1 assays were performed on brains as previously described (Macauley et al., 2012). The values were normalized to total protein measured using a Coomassie dye-binding assay (Bio-Rad Laboratories). One-way ANOVA and Tukey's multiple-comparison tests were used to determine significance.

Brain atrophy. At 7.5 months, treated and sham-treated mice $(n=$ 3-9) were killed and the brains dissected by a researcher blinded to both age and genotype as previously described (Macauley et al., 2011). Each brain was weighed and differences in brain weights were analyzed with one-way ANOVAs followed by Bonferroni correction post hoc tests.

Brain processing, Nissl staining, and regional volume measurements. At 7.5 months of age, brains from treated and sham-treated mice $(n=4$ mice/group) were removed and fixed for $48 \mathrm{~h}$ in $4 \%$ paraformaldehyde in PBS followed by cryoprotection in a $30 \%$ sucrose solution. One hemisphere of each forebrain and the cerebellum were sectioned on a freezing microtome at $40 \mu \mathrm{m}$. For Nissl staining, sections were mounted onto coated SuperFrost microscope slides, allowed to air dry overnight, and stained with a $0.05 \%$ cresyl fast violet solution. Unbiased estimates of the volumes of cortex, hippocampus, striatum, and thalamus were obtained via the Cavalieri method as previously described. Statistical differences were determined using one-way ANOVAs followed by Bonferroni correction post hoc tests.

Immunohistochemistry for glial markers. Immunostaining and quantification for GFAP and CD68 were performed in regions of interest as previously reported (Macauley et al., 2012). One-way ANOVAs with Bonferroni correction post hoc tests were used to determine statistical significance.

Analysis of cytokine profiles. To quantify the concentration of chemokines and cytokines within the brain ( $n=3-4$ mice per group), a 14biomarker Multi-Analyte Profile was generated for 7.5-month-old treated and sham-treated mice by Rules Based Medicine using standard Luminex Technology (Luminex ) for 14 separate analytes as previously described (Macauley et al., 2011). One-way ANOVA followed by Bonferroni post hoc tests was used to determine significant changes.

\section{Results \\ Life span}

One measure of therapeutic efficacy is longevity. $\mathrm{Ppt1^{-/- }}$ mice die at $\sim 34.9$ weeks compared with normal littermates (Fig. 1A). Daily injections of MW151 alone did not significantly increase life span compared with sham-treated $P p t 1^{-/-}$mice (35.4 vs 34.9 weeks, respectively). However, treatment with AAV alone or $\mathrm{AAV}+\mathrm{MW} 151$ injections led to significant increases in median survival. Moreover, AAV + MW151 treatment resulted in a significant increase in life span compared with AAV alone (47 vs 45.4 weeks, respectively). 
A

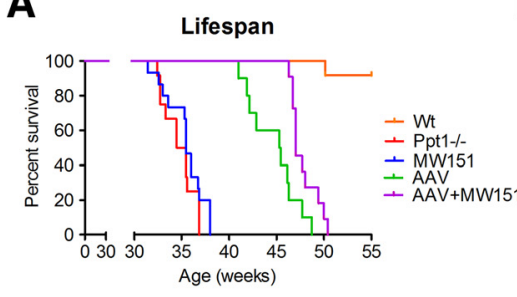

B

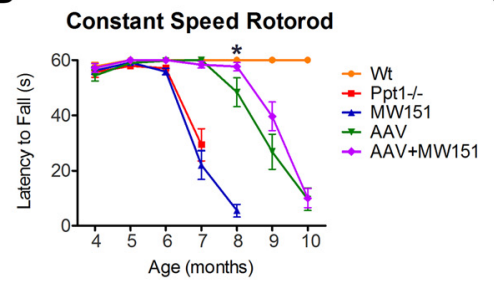

C

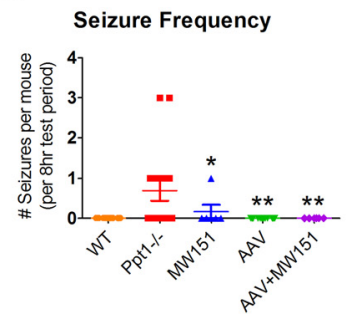

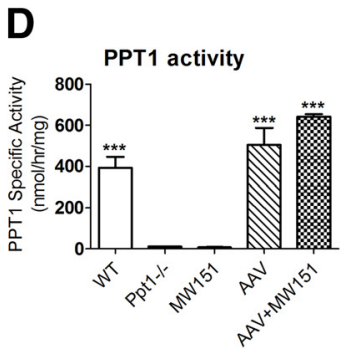

Figure 1. A, Increased median life span was observed with treatment of AAV only or AAV + MW151 in Ppt1 ${ }^{-1-}$ mice. No improvement in life span was seen with MW151 alone. $\boldsymbol{B}, \mathrm{AAV}$ alone significantly improved performance on the constant-speed rotarod test. AAV + MW151 provided a further increase in motor performance at 8 months of age. C, Treatment with MW151 alone, AAV alone, or the combination, decreased seizure frequency in $P p t 1^{-1-}$ mice. Untreated $P p t 1^{-1-}$ mice $(n=16)$ had a total of 11 seizures during the monitoring period, while no WT mice $(n=20)$ had seizures. Ppt $1^{-\prime-}$ mice treated with MW151 only $(n=6)$ had a single seizure during the same monitoring period. Moreover, AAV only $(n=24)$, or AAV in combination with MW151 $(n=6)$, eradicated this seizure phenotype. $\mathbf{D}$, Compared withPpt ${ }^{-1}{ }^{-}$brains, there is a significant increase in PPT1 activity following treatment with AAV only or AAV + MW151. Daily injections of MW151 did not increase PPT1 activity $\left({ }^{*} p<0.05 ;{ }^{* *} p<0.01 ;{ }^{* * *} p<0.001\right)$. Error bars represent \pm SEM.
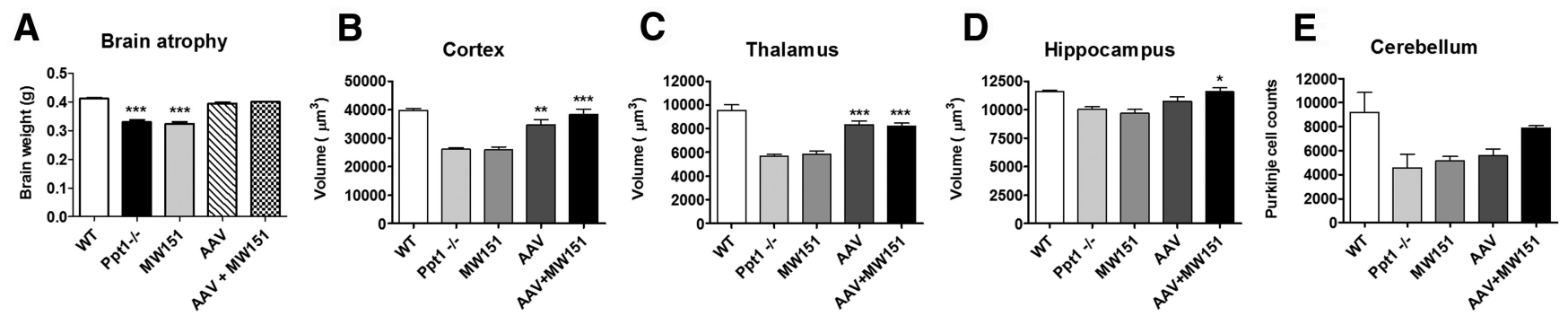

Figure 2. A, Brain weight, a marker of CNS atrophy, increased in Ppt $1^{-/-}$mice following therapeutic intervention with AAV only or AAV + MW151 compared with untreated Ppt $1^{-/-}$mice. $\boldsymbol{B}$, Cortical and thalamic volumes (C) increased significantly following AAV-only or AAV + MW151 therapy. $\boldsymbol{D}$, Hippocampal volume was significantly increased following AAV + MW151 therapy. $\boldsymbol{E}$, There was no significant increase in cerebellar volume after therapeutic intervention $\left({ }^{*} p<0.05 ;{ }^{* *} p<0.01 ;{ }^{* * *} p<0.001\right)$. Error bars represent \pm SEM.

\section{Constant speed rotarod}

We investigated the combined effect of AAV-mediated gene therapy and daily MW151 injections on motor deficits in the Ppt1 ${ }^{-/-}$ mouse (Fig. 1B). At 4 months, all treated and sham-treated mice mastered the constant speed paradigm and performed similar to WT. Beginning at 6 months, motor function in sham-treated and MW151-only Ppt1 ${ }^{-1-}$ mice deteriorated when compared with AAV-only, AAV + MW151, and WT mice. The performance of $\mathrm{Ppt1}^{-1-}$ or MW151-only mice continued to decline until their death at 7 and 8 months., respectively. In contrast, mice treated with AAV only or AAV + MW151 performed comparably to normal littermates through 7 months. At 8 months, the AAV + MW151 group performed significantly $(p<0.05)$ better than the AAV-only group. The performance of AAV-only and AAV + MW151-treated mice continued to deteriorate at 9 months, culminating in a nadir in performance at 10 months.

\section{Seizure activity}

Using a force plate actometer combined with video monitoring, seizure activity was examined in treated and sham-treated mice (Fig. 1C). At 7.5 months, $43.8 \%$ of $\mathrm{Ppt1}^{-1-}$ mice had at least one spontaneous seizure during the $8 \mathrm{~h}$ monitoring session. In contrast, no spontaneous seizure activity was observed in WT mice. Ppt1-deficient mice treated with MW151 alone displayed decreased seizure activity, where only 1 of 6 mice tested had seizures. Treatment with AAV only or AAV + MW151 eradicated the seizure phenotype.

\section{PPT1 activity}

PPT1 activity in the normal brain is $\sim 393.2 \mathrm{nmol} / \mathrm{mg} / \mathrm{h}$ (Fig. $1 D)$. In contrast, PPT1 activity was significantly decreased in the brains of untreated $P \mathrm{Pt}^{-1-}$ and MW151-treated mice (10.8 and
$8.5 \mathrm{nmol} / \mathrm{mg} / \mathrm{h}$, respectively). Following AAV-only or AAV + MW151 treatment, there is a significant increase in PPT1 activity to 504.1 and $640.3 \mathrm{nmol} / \mathrm{mg} / \mathrm{h}$, respectively.

\section{Overall brain atrophy}

Brain weight measurements were used as a simple means to determine overall brain atrophy in treated and untreated mice (Fig. $2 A)$. In the $\mathrm{Ppt1}^{-1-}$ brain, there was a significant decrease in brain weight, by $\sim 20 \%$, at 7.5 months compared with WT mice $(p<0.001)$. Treatment with MW151 alone had no effect on overall brain atrophy. In contrast, the brain weights from AAVonly and AAV + MW151 groups were significantly increased compared with untreated $P p t 1^{-1-}$ controls and were comparable to WT.

\section{Regional atrophy and neuronal loss}

There was a significant reduction in regional volume within the cortex, hippocampus, thalamus, and cerebellum of $P p t 1^{-1-}$ mice compared with WT (Fig. 2B-E). Daily injections of MW151 did not alter regional atrophy in any of the regions assayed. In contrast, treatments including intracranial delivery of AAV or AAV + MW151 resulted in significant increases in cortical and thalamic volume. Interestingly, the combination of AAV + MW151 significantly increased hippocampal volume $(p<0.05)$ while treatment with AAV alone did not. There was a significant decrease in calbindin-positive Purkinje cells within the cerebellum of $P p t 1^{-\prime-}$ mice. Treatment with MW151 alone did not decrease neuronal loss within the cerebellum. Although treatment with AAV or AAV + MW151 did not significantly increase Purkinje cell number, there appeared to be an increase in Purkinje cell number in the AAV + MW151 group that did not reach significance. 
A
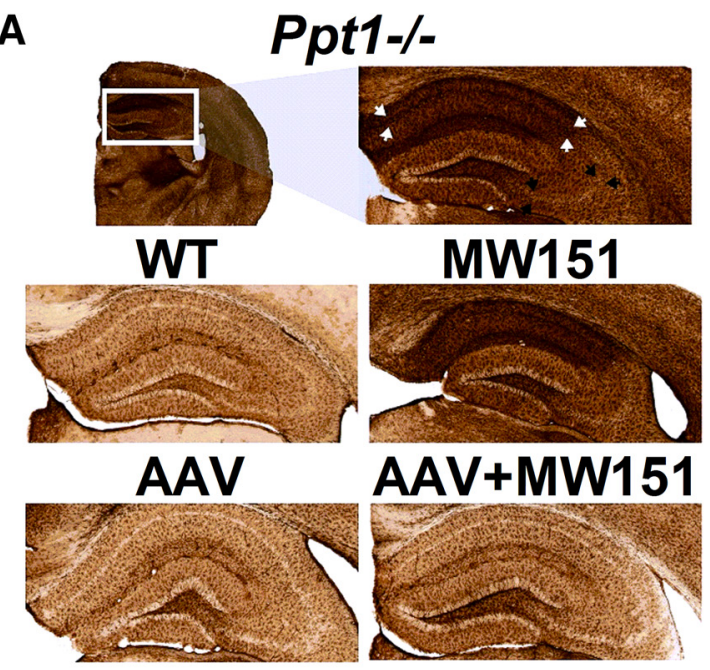

c
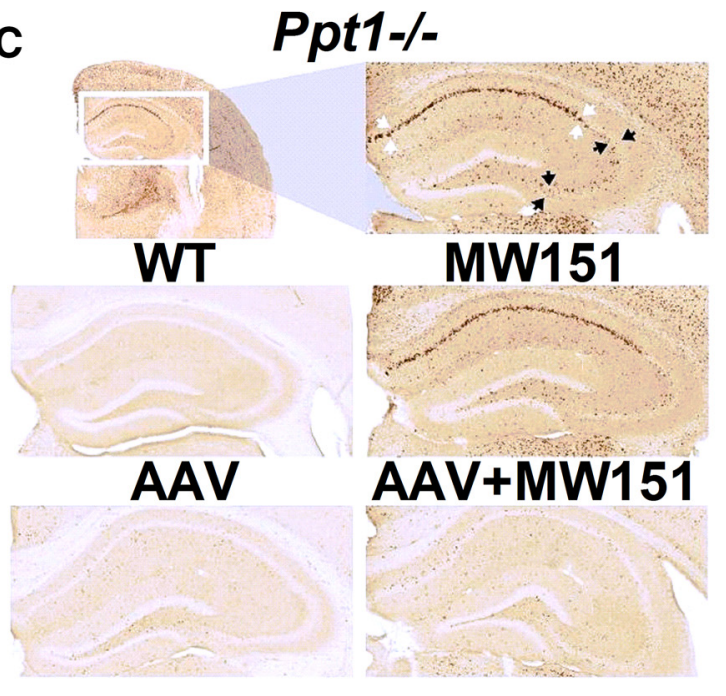

B

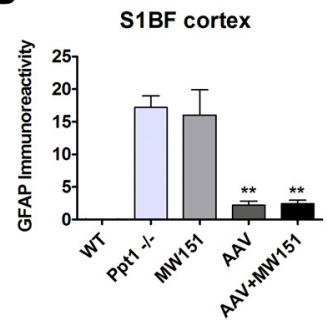

Hippocampus (CA1 field)

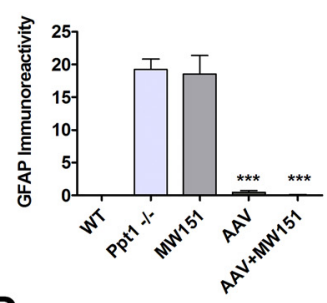

D
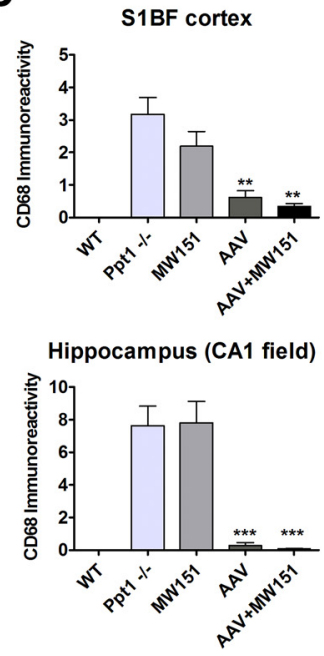

Thalamus (VPM/VPL)

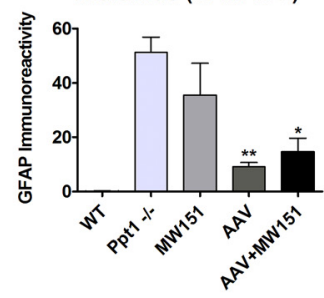

Cerebellum (Lobe VIII)
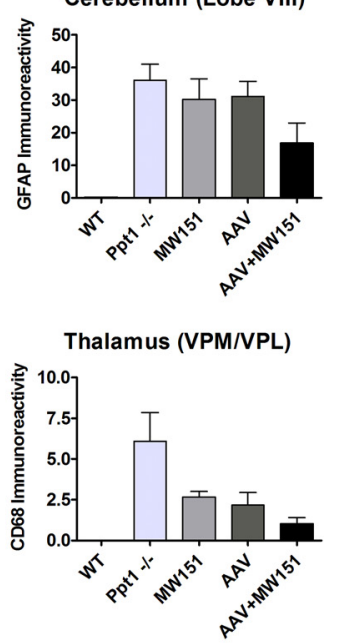

Cerebellum (Lobe VIII)

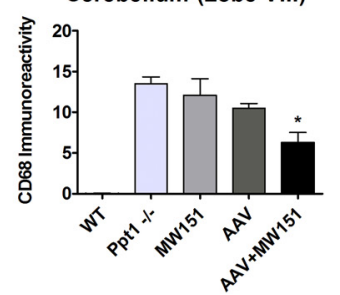

Figure 3. Astrocyte (GFAP) and microglial (CD68) activation in the $P p t 1^{-1-}$ brain following therapeutic intervention. There were significant increases in astrocyte $(\boldsymbol{A}, \boldsymbol{B})$ and microglia (C, D) staining in the S1BF cortex, the ventral posteromedial (VPM)/ventral posterolateral (VPL) region of the thalamus, CA1 field of the hippocampus, and lobe VIII of the cerebellum in Ppt $1^{-1-}$ mice. B, Following AAV alone or AAV + MW151 treatment, there was a significant decrease in GFAP staining within the cortex, thalamus, and hippocampus, but not in the cerebellum. Treatment with MW151 alone had little effect on GFAP immunoreactivity. C, There was a significant decrease in microglial activation in the cortex and hippocampus of Ppt $1^{-1-}$ mice following AAV or AAV + MW151 therapy. The combination of AAV + MW151 also significantly decreased CD68 staining in the cerebellum. There was a trend toward a decrease in CD68 staining in the MW151-only group in the cortex and thalamus, but this did not reach significance $\left({ }^{*} p<0.05 ;{ }^{* *} p<0.01 ;{ }^{* *} p<0.001\right)$. Error bars represent \pm SEM.

\section{Regional neuroinflammation}

Astrocyte activation (increased GFAP expression; Fig. $3 A, B$ ) and microglial reactivity (increased CD68 expression; Fig. $3 C, D$ ) are key components of INCL neuropathology. There is a significant increase in astrocyte activation at 7.5 months in the cortex, thalamus, hippocampus, and cerebellum of $P p t 1^{-1-}$ mice compared with WT controls (Fig. 3B). Treatment with MW151 alone did not decrease astrocyte activation in any of the brain regions assayed. However, treatment with AAV or AAV + MW151 significantly decreased astrocyte activation within cortex, thalamus, and hippocampus. Interestingly, there was no effect observed with either treatment in the cerebellum.

Profound microglial reactivity is observed within the $P p t 1^{-/-}$ cortex, thalamus, hippocampus, and cerebellum at 7.5 months (Fig. 3C,D). Following treatment with MW151 only, there appeared to be a decrease in CD68 staining within the cortex, although this was not significant. Treatment with AAV and AAV + MW151 resulted in a significant decrease $(p<0.01)$ in microglial activation within the cortex and hippocampus. Similarly, CD68 immunostaining appeared to decrease in the thalamus of mice treated with MW151 alone, AAV only, or AAV + MW151, but this effect did not reach significance. Also, combination therapy with AAV + MW151 resulted in a significant decrease in microglial activation in $P p t 1^{-1-}$ cerebellum $(p<0.05)$, while AAV alone had little effect.

\section{Cytokine production}

Previous reports describe a significant increase in cytokine expression in $P p t 1^{-\prime-}$ brains as disease progresses (Qiao et al., 2007; Kielar et al., 2009; Macauley et al., 2011). Therefore, we sought to measure the levels of various pro-inflammatory markers such as IFN- $\gamma$ and TNF- $\alpha$ (Fig. 4A); lymphocyte/ monocyte mediators, such as Oncostatin M and IP-10 (Fig. $4 B$ ); and monocyte mediators, such as MIP- $1 \beta$, MIP-2, and MCP-1 (Fig. 4C). At 7.5 months, pro-inflammatory cytokines as well as monocyte and lymphocyte mediators are elevated in $P p t 1^{-1-}$ mice compared with littermate controls. Although no significant effects on cytokine levels were observed with any 
A

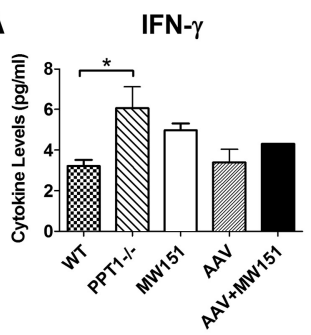

C

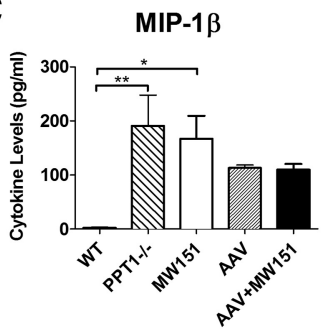

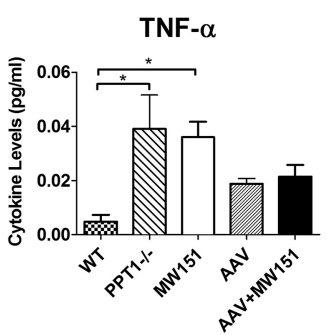

B

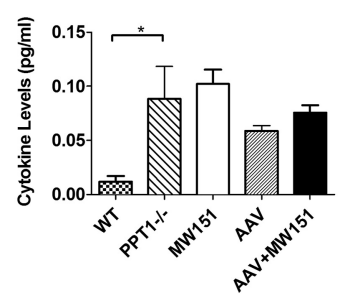

MIP-2

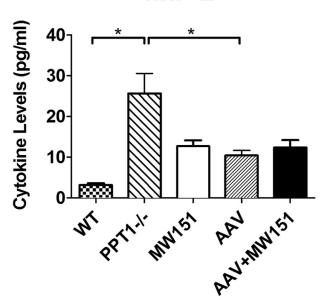

IP-10

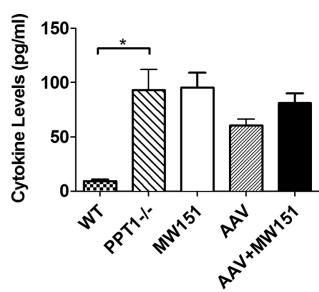

MCP-1

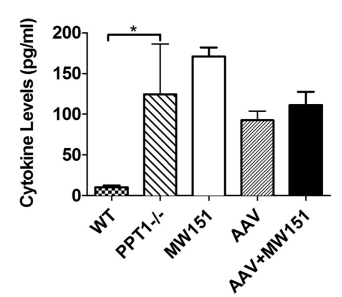

Figure 4. Therapeutic intervention decreases cytokine production in the $P p t 1^{-1-}$ brains. Luminex assays were performed for $(\boldsymbol{A})$ pro-inflammatory markers, $(\boldsymbol{B})$ lymphocyte/monocyte activators, and (C) monocyte activators. Elevations in the cytokines, IFN- $\gamma$, TNF- $\alpha$, MIP-1B, MIP-2, MCP-1, 0ncostatin M, and IP-10, were observed in Ppt $1^{-1-}$ mice. Treatment with AAV only and AAV + MW151 produced a trend toward a decrease in IFN- $\gamma$, TNF- $\alpha$, MIP-1B, MIP-2, MCP-1, Oncostatin M, and IP-10, although the change was not statistically significant. Error bars represent \pm SEM.

treatment, all treatments appeared to decrease levels within the brain. Specifically, AAV-only and AAV + MW151 groups appeared to have more robust effects on all cytokine assays. However, the MW151-alone appeared to decrease several monocyte (MIP-2 and MCP-1) and monocyte/leukocyte (Oncostatin M) mediators; however, this did not result in statistical significance.

\section{Discussion}

Previous studies in the $P p t 1^{-/-}$mice demonstrated that neuroinflammation is central to the pathogenesis of INCL (Kielar et al., 2007; Macauley et al., 2009, 2011). Widespread astrocytosis and microglial activation accompany this frank neurodegenerative disorder. Before the onset of neurodegeneration, astrocyte activation occurs in specific brain regions that ultimately suffer neuronal loss and brain atrophy. In addition to astrocytosis, microglial activation occurs before and during neuronal cell loss. Concurrent with glial activation, the $P p t 1^{-/-}$brain displays elevated levels of chemokines and cytokines, which are responsible for the recruitment of monocytes and leukocytes into the CNS. Recent studies also demonstrated that inactivation of the adaptive immune system in $P p t 1^{-1-}$ mice spared axonal damage and neuronal loss (Groh et al., 2013). Therefore, increased expression of cytokines within the CNS and the infiltration of peripheral immune cells are closely associated with the neuronal pathology and functional decline. Given the evidence implicating pro-inflammatory cytokine-mediated neuroinflammation in $P p t 1^{-1-}$ mice, we investigated the therapeutic value of MW151, a small molecule capable of mitigating glial activation and cytokine production, as a cotherapeutic approach with CNS-directed, AAV-mediated gene therapy in the mouse model of INCL (Macauley et al., 2012; Roberts et al., 2012).

AAV-mediated gene therapy alone resulted in significant biochemical, histological, and behavioral improvements in PPT1deficient mice. The unexpected main positive finding of MW151 treatment alone was the significant decrease in seizure incidence in the Ppt1 ${ }^{-1-}$ mice compared with untreated controls. This is an important finding given the severity of the seizure phenotype in INCL. In addition, this suggests that seizures associated with
INCL might be related to the neuroinflammatory response associated with neuronal dysfunction. However, MW151 did not decrease histopathological markers of disease. This is not entirely surprising since MW151 does not target the primary defect in $P p t 1^{-/-}$mice, an enzyme deficiency which results in accumulation of undegraded material. Long-term treatment (every day for nearly 1 year) with MW151 at $2.5 \mathrm{mg} / \mathrm{kg}$ was well tolerated but might not be sufficient given the severity of disease progression. It is possible that chronic treatment with even higher doses would be well tolerated and perhaps result in greater benefit alone or in combination with gene therapy. A dose-ranging study showed that chronic administration of MW151 at doses $>100$-fold higher than those reported here were well tolerated and resulted in little or no toxicity (D.M. Watterson, personal communication).

In previous studies (Macauley et al., 2011; Hu et al., 2012; Roberts et al., 2012) that target the primary defect such as CNS-directed gene therapy or ERT, clinical improvement was modest but improved with use of adjunct therapies. This is likely due to the aggressive and complex nature of this disease. INCL is a chronic disease with a rapid clinical course, affecting the brain in its entirety. This poses a unique set of challenges for replacement therapy. Only addressing the enzymatic deficit in INCL patients will likely yield partial results. However, when used in combination with drugs such as MW151, there is the additional benefit of targeting a secondary pathogenic mechanism in conjunction with the appeal of less invasive dosing. Beyond INCL, this has implications for treating other forms of Batten disease, such as late-infantile and juvenile NCL, given that glial activation and seizure deficits are prominent features of these diseases as well (Oswald et al., 2005; Chang et al., 2008; Chen et al., 2009; Weimer et al., 2009). The concept of combining replacement therapy with smallmolecule cotherapy is also gaining acceptance in other areas of neurotherapeutics (Glover et al., 2012). Overall, the results reported here demonstrate the potential of using AAV-mediated enzyme replacement therapy combined with mechanism-based, small-molecule drug treatment for INCL 


\section{References}

Bachstetter AD, Norris CM, Sompol P, Wilcock DM, Goulding D, Neltner JH, St Clair D, Watterson DM, Van Eldik LJ (2012) Early stage drug treatment that normalizes proinflammatory cytokine production attenuates synaptic dysfunction in a mouse model that exhibits age-dependent progression of Alzheimer's disease-related pathology. J Neurosci 32: 10201-10210. CrossRef Medline

Chang M, Cooper JD, Sleat DE, Cheng SH, Dodge JC, Passini MA, Lobel P, Davidson BL (2008) Intraventricular enzyme replacement improves disease phenotypes in a mouse model of late infantile neuronal ceroid lipofuscinosis. Mol Ther 16:649-656. CrossRef Medline

Chen YH, Chang M, Davidson BL (2009) Molecular signatures of disease brain endothelia provide new sites for CNS-directed enzyme therapy. Nat Med 15:1215-1218. CrossRef Medline

Chrzaszcz M, Venkatesan C, Dragisic T, Watterson DM, Wainwright MS (2010) Minozac treatment prevents increased seizure susceptibility in a mouse "two-hit" model of closed skull traumatic brain injury and electroconvulsive shock-induced seizures. J Neurotrauma 27:1283-1295. CrossRef Medline

Glover LE, Tajiri N, Weinbren NL, Ishikawa H, Shinozuka K, Kaneko Y, Watterson DM, Borlongan CV (2012) A step-up approach for cell therapy in stroke: translational hurdles of bone marrow-derived stem cells. Transl Stroke Res 3:90-98. CrossRef Medline

Griffey MA, Wozniak D, Wong M, Bible E, Johnson K, Rothman SM, Wentz AE, Cooper JD, Sands MS (2006) CNS-directed AAV2-mediated gene therapy ameliorates functional deficits in a murine model of infantile neuronal ceroid lipofuscinosis. Mol Ther 13:538-547. CrossRef Medline

Griffey M, Bible E, Vogler C, Levy B, Gupta P, Cooper J, Sands MS (2004) Adeno-associated virus 2-mediated gene therapy decreases autofluorescent storage material and increases brain mass in a murine model of infantile neuronal ceroid lipofuscinosis. Neurobiol Dis 16:360-369. CrossRef Medline

Groh J, Kühl TG, Ip CW, Nelvagal HR, Sri S, Duckett S, Mirza M, Langmann $\mathrm{T}$, Cooper JD, Martini R (2013) Immune cells perturb axons and impair neuronal survival in a mouse model of infantile neuronal ceroid lipofuscinosis. Brain 136:1083-1101. CrossRef Medline

Gupta P, Soyombo AA, Atashband A, Wisniewski KE, Shelton JM, Richardson JA, Hammer RE, Hofmann SL (2001) Disruption of PPT1 or PPT2 causes neuronal ceroid lipofuscinosis in knockout mice. Proc Natl Acad Sci U S A 98:13566-13571. CrossRef Medline

Hofmann SL, Das AK, Yi W, Lu JY, Wisniewski KE (1999) Genotypephenotype correlations in neuronal ceroid lipofuscinosis due to palmitoyl-protein thioesterase deficiency. Mol Genet Metab 66:234-239. CrossRef Medline

Hu J, Lu JY, Wong AM, Hynan LS, Birnbaum SG, Yilmaz DS, Streit BM, Lenartowicz EM, Thompson TC, Cooper JD, Hofmann SL (2012) Intravenous high-dose enzyme replacement therapy with recombinant palmitoyl-protein thioesterase reduces visceral lysosomal storage and modestly prolongs survival in a preclinical mouse model of infantile neuronal ceroid lipofuscinosis. Mol Genet Metab 107:213-221. CrossRef Medline

Hu W, Ralay Ranaivo H, Roy SM, Behanna HA, Wing LK, Munoz L, Guo L, Van Eldik LJ, Watterson DM (2007) Development of a novel therapeutic suppressor of brain proinflammatory cytokine up-regulation that at- tenuates synaptic dysfunction and behavioral deficits. Bioorg Med Chem Lett 17:414-418. CrossRef Medline

Karpus WJ, Reynolds N, Behanna HA, Van Eldik LJ, Watterson DM (2008) Inhibition of experimental autoimmune encephalomyelitis by a novel small molecular weight proinflammatory cytokine suppressing drug. J Neuroimmunol 203:73-78. CrossRef Medline

Kielar C, Maddox L, Bible E, Pontikis CC, Macauley SL, Griffey MA, Wong M, Sands MS, Cooper JD (2007) Successive neuron loss in the thalamus and cortex in a mouse model of infantile neuronal ceroid lipofuscinosis. Neurobiol Dis 25:150-162. CrossRef Medline

Kielar C, Wishart TM, Palmer A, Dihanich S, Wong AM, Macauley SL, Chan CH, Sands MS, Pearce DA, Cooper JD, Gillingwater TH (2009) Molecular correlates of axonal and synaptic pathology in mouse models of Batten disease. Hum Mol Genet 18:4066-4080. CrossRef Medline

Macauley SL, Wozniak DF, Kielar C, Tan Y, Cooper JD, Sands MS (2009) Cerebellar pathology and motor deficits in the palmitoyl protein thioesterase 1-deficient mouse. Exp Neurol 217:124-135. CrossRef Medline

Macauley SL, Pekny M, Sands MS (2011) The role of attenuated astrocyte activation in infantile neuronal ceroid lipofuscinosis. J Neurosci 31: 15575-15585. CrossRef Medline

Macauley SL, Roberts MS, Wong AM, McSloy F, Reddy AS, Cooper JD, Sands MS (2012) Synergistic effects of central nervous system-directed gene therapy and bone marrow transplantation in the murine model of infantile neuronal ceroid lipofuscinosis. Ann Neurol 71:797-804. CrossRef Medline

Oswald MJ, Palmer DN, Kay GW, Shemilt SJ, Rezaie P, Cooper JD (2005) Glial activation spreads from specific cerebral foci and precedes neurodegeneration in presymptomatic ovine neuronal ceroid lipofuscinosis (CLN6). Neurobiol Dis 20:49-63. CrossRef Medline

Qiao X, Lu JY, Hofmann SL (2007) Gene expression profiling in a mouse model of infantile neuronal ceroid lipofuscinosis reveals upregulation of immediate early genes and mediators of the inflammatory response. BMC Neurosci 8:95. CrossRef Medline

Reddy AS, Kim JH, Hawkins-Salsbury JA, Macauley SL, Tracy ET, Vogler CA, Han X, Song S-K, Wozniak DF, Fowler SC, Klein RS, Sands MS (2011) Bone marrow transplantation augments the effect of brain and spinal cord-directed adeno-associated virus $2 / 5$ gene therapy by altering inflammation in the murine model of globoid-cell leukodystrophy. J Neurosci 31:9945-9957. CrossRef Medline

Roberts MS, Macauley SL, Wong AM, Yilmas D, Hohm S, Cooper JD, Sands MS (2012) Combination small molecule PPT1 mimetic and CNSdirected gene therapy as a treatment for infantile neuronal ceroid lipofuscinosis. J Inherit Metab Dis 35:847-857. CrossRef Medline

Somera-Molina KC, Robin B, Somera CA, Anderson C, Stine C, Koh S, Behanna HA, Van Eldik LJ, Watterson DM, Wainwright MS (2007) Glial activation links early life seizures and long-term neurologic dysfunction: evidence using a small molecule inhibitor of proinflammatory cytokine upregulation. Epilepsia 48:1785-1800. CrossRef Medline

Vesa J, Hellsten E, Verkruyse LA, Camp LA, Rapola J, Santavuori P, Hofmann SL, Peltonen L (1995) Mutations in the palmitoyl protein thioesterase gene causing infantile neuronal ceroid lipofuscinosis. Nature 376:584587. CrossRef Medline

Weimer JM, Benedict JW, Getty AL, Pontikis CC, Lim MJ, Cooper JD, Pearce DA (2009) Cerebellar defects in a mouse model of juvenile neuronal ceroid lipofuscinosis. Brain Res 1266:93-107. CrossRef Medline 